\title{
Investigation of nitrate transformation in the vadose zone: laboratory experimental studies
}

\author{
SEUNGHAK LEE ${ }^{1,2}$, SungJIK OH ${ }^{1,2}$, SAEROM PARK $^{3}$
}

${ }^{1}$ Water Cycle Research Center, National Agenda Research Division, Korea Institute of Science and Technology (KIST), Seoul 02792, South Korea

${ }^{2}$ Division of Energy and Environmental Technology, KIST School, Korea University of Science and Technology (UST), Seoul 02792, South Korea

${ }^{3}$ Urban Water Circulation Research Center, Department of Land, Water and Environment Research, Korea Institute of Civil Engineering and Building Technology (KICT), Gyeonggi-do 10223, South Korea

Even though lots of studies have been done for understanding the nitrate (NO3-) behaviors in the subsurface under saturated condition, little is known about the NO3- fate in the vadose zone (VZ). The VZ extending from the ground surface to the groundwater table is described as the partially saturated porous medium. Thus, during the passage through VZ, NO3might be exposed to various redox conditions having different oxygen and water contents. In this study, the possible transformation of NO3- in the VZ was investigated by laboratory experiments. Different shapes of subsurface porous networks were simulated by using different soil textures including sandy loams, loamy sands and sands. Batch experiments were conducted for investigating the reactions at a range of water contents in different soil textures, which might allow us to expect the NO3- fate under locally and temporarily steady conditions. For simulating the reactions under continuously varying redox conditions, soil column experiments were conducted with setting up the groundwater table at the bottom and applying the intermittent precipitation at the top. Batch and column experiments are currently under way, and the results will be presented at the conference. 